\title{
Comparison of hard X-ray spectra of luminous radio galaxies and their radio-quiet counterparts
}

\author{
Maitrayee Gupta, ${ }^{1 \star}$ Marek Sikora, ${ }^{1}$ Katarzyna Rusinek ${ }^{1}$ and Greg M. Madejski ${ }^{2}$ \\ ${ }^{1}$ Nicolaus Copernicus Astronomical Center, Bartycka 18, 00-716 Warsaw, Poland \\ ${ }^{2}$ Kavli Institute for Particle Astrophysics and Cosmology, Stanford University, Stanford, CA 94305, USA
}

Accepted XXX. Received YYY; in original form ZZZ

\begin{abstract}
We study the differences in X-ray properties of luminous radio galaxies and their radio-quiet counterparts. In order to avoid biases associated with the dependence of $\mathrm{X}$-ray properties on the black hole mass and Eddington ratio, the radio-loud and radioquiet objects are selected to cover similar ranges of these parameters. Our studies are based on the X-ray data from the Swift/BAT catalogue. We confirm previous results that radio galaxies are on average X-ray-louder than radio-quiet AGNs, but find that their spectral slopes are very similar. This suggests that in radio-loud and radio-quiet AGNs the hard X-rays are produced in the same region and by the same mechanism. We argue that this region can be associated with the hot, geometrically thick, central portion of the accretion flow, where production of hard X-rays is likely to be dominated by Comptonization of the optical/UV radiation of the truncated 'cold' accretion disc by hot electrons. The larger X-ray luminosities in radio-loud AGNs may result from larger radiative efficiencies of the innermost portions of the accretion flows around faster rotating black holes.
\end{abstract}

Key words: quasars - galaxies: jets — radiation mechanisms: non-thermal — acceleration of particles

\section{INTRODUCTION}

Known already since the 1980 s, the so-called radiodichotomy of AGNs Strittmatter et al. 1980, Peacock et al. 1986, Kellermann et al. 1989 |Miller et al. 1990) is still awaiting theoretical explanation. Their radio loudness parameter, defined as the ratio of radio luminosity of associated with them radio sources to optical luminosity of their accretion discs, spans several orders of magnitude (e.g. Ivezić et al. 2002, White et al. 2007; Baloković et al. 2012 Kratzer \& Richards 2015), and this implies similarly large range of the jet production efficiency (e.g. Sikora \& Begelman 2013). Most luminous radio sources need to be powered by jets at rates approaching or sometimes even exceeding accretion luminosities (e.g. Rawlings \& Saunders 1991, Punsly 2007,

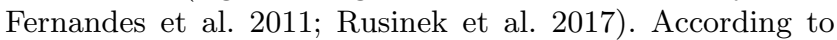
recent theoretical and numerical studies, the production of such powerful jets can be via the Blandford-Znajek mechanism (Blandford \& Znajek 1977) involving a fast rotating black hole immersed in very strong magnetic field. Such field is found to be maximized in the model called magneticallyarrested-disc (MAD) scenario, in which magnetic flux is

^ E-mail: mgupta@camk.edu.pl confined on the BH by ram pressure of the accretion flow (Narayan et al. 2003 Igumenshchev 2008, Punsly et al. 2009. Tchekhovskoy et al. 2011| McKinney et al. 2012). If that is true, one might expect that structure and physics of accretion flows in such objects may differ significantly from those in radio-weak AGNs. However their averaged SEDs (spectral energy distributions) in IR, optical and UV bands are very similar (Elvis et al. 1994, de Vries et al. 2006, Richards et al. 2006 Shang et al. 2011| Gupta et al. 2016), and statistically significant differences are found only in X-ray bands. Specifically, RL quasars have been found to be X-ray-louder and to have harder X-ray spectra than RQ quasars (Zamorani et al. 1981, Worrall et al. 1987, Wilkes \& Elvis 1987). These results were confirmed also for lower-redshift type 1 AGNs represented by broad-line radio galaxies (BLRGs) and radioquiet (RQ) Seyfert 1 galaxies (Woźniak et al. 1998, Sambruna et al. 1999, Grandi et al. 2006 Kataoka et al. 2011. Miller et al. 2011). In addition, it was found that X-ray reflection features are weaker in radio-loud (RL) than in RQ AGNs, and this led to speculations that it is so because in RL AGNs the X-ray emission is contributed not only by the disc corona, as originally proposed by Liang \& Thompson (1979) and Haardt \& Maraschi (1991), but also by the base of a jet (e.g. Woźniak et al. 1998). An alternative possibil- 
ity was that accretion discs in luminous RL AGNs could be on average more ionized than in Seyferts (Ballantyne et al. 2002 Evans et al. 2010, Ballantyne et al. 2014) or that geometrically thin and optically thick accretion discs in such AGNs are truncated at some radius, and that X-rays are emitted by the hot ADAF (Advection Dominated Accretion Flow) formed within that radius (e.g. Narayan \& Yi 1994. Yuan \& Narayan 2014). The latter possibility was specifically explored following detailed observations of individual BLRGs in different X-ray bands (e.g. Eracleous et al. 2000, Grandi \& Palumbo 2007; Sambruna et al. 2009| Tazaki et al. 2010 Cowperthwaite \& Reynolds 2012 Lohfink et al. 2013). However, all those comparisons of X-ray properties of $\mathrm{RL}$ and RQ AGNs were performed selecting them using different methods and/or having their samples with very different average Eddington ratios (usually significantly lower in RL AGNs than in RQ ones) and BH masses (typically much larger in RL AGNs than in RQ ones, e.g. McLure \& Jarvis 2004 Chiaberge \& Marconi 2011. Koziel-Wierzbowska et al. 2017).

Currently, with much larger samples of AGNs detected in the hard X-ray band as provided by the Swift/BAT all-sky surveys (Baumgartner et al. 2013, Oh et al. 2018) it became possible to study the differences between X-ray spectra in RL and RQ AGNs using their subsamples covering similar ranges of $\mathrm{BH}$ masses and Eddington ratios. Furthermore, by using the mid-IR rather than optical data to estimate AGN bolometric luminosities, we expanded the compared samples by including both type 1 and type 2 AGNs, but reduced them to cover the ranges of $\mathrm{BH}$ masses and Eddington ratios typical for luminous FR II radio galaxies (Fanaroff \& Riley 1974). The resulting subsamples are represented by 24 broad-line and narrow-line radio galaxies, and by 46 broadline and narrow-line radio-quiet Seyfert galaxies.

The paper is organized as follows: in Sections 2 and 3 we briefly describe our selection procedure and data processing. In Section 4 we present the results of our comparison analysis. In Section 5 we provide possible interpretation of these results and in Section 6 we summarize our findings.

Throughout the paper we assume a $\Lambda$ CDM cosmology with $H_{0}=70 \mathrm{~km} \mathrm{~s}^{-1} \mathrm{Mpc}^{-1}, \Omega_{m}=0.3$, and $\Omega_{\Lambda}=0.70$.

\section{THE DATA AND SAMPLES}

\subsection{X-ray data}

The Swift/Burst Alert Telescope (BAT) has carried out an all-sky survey in the hard X-ray range (14-195 keV) and in the first 70 months of observation, it catalogued 1210 objects (Baumgartner et al. 2013). The primary sample of AGNs used in our research is derived from the BAT AGN Spectroscopic Survey (BASS), from the studies conducted by Ricci et al. (2017) where authors presented X-ray spectral parameters for 838 AGNs.

Ricci et al. (2017) also used data from Swift/XRT, $X M M-$ Newton, ASCA, Chandra, and Suzaku observations at energies $\leq 10 \mathrm{keV}$ in order to derive the column density and reflection features of Swift/BAT AGNs. Using their data on column density we reduced the sample to include only the Compton-thin AGNs $\left(\log N_{H}<24\right)$ therefore excluding objects with significantly absorbed hard X-rays. This criterion restricted our sample to 776 sources.
Our next step was to reject objects classified as blazars, since X-rays from these objects are likely to be dominated by the Doppler-boosted radiation from relativistic jets pointing towards us rather than by accretion flows. The blazars were selected by using the Ricci et al. (2017) catalogue, where they were identified by cross-matching of Swift/BAT catalogue with the Roma-BZCAT 26 catalogue of blazars (Massaro et al. 2015). Rejection of blazars left us with 664 objects.

\subsection{Radio data}

Radio data for our sample, which includes sources from the Northern as well as Southern hemisphere, were collected from two radio catalogues: National Radio Astronomy Observatory (NRAO) Very Large Array (VLA) Sky Survey (NVSS; Condon et al. 1998) and Sydney University Molonglo Sky Survey (SUMSS; Bock et al. 1999; Mauch et al. 2003). NVSS has been carried out at $1.4 \mathrm{GHz}$ while SUMSS data is given at $843 \mathrm{MHz}$. Both these surveys have similar angular resolution (45 arcsec FWHM for NVSS and $45 \times 45 \operatorname{cosec}|\delta| \operatorname{arcsec}^{2}$ for SUMSS) and sensitivities $(\sim 2.5$ mJy).

Since AGNs can have multiple radio sources associated with them, we ensured to account for various types of morphologies (single or multiple, e.g. double and triple, matches). Below we describe our method in more detail.

As the first step in finding the NVSS associations for the objects in our sample we decided to use a matching radius of 3 arcmin from the optical coordinates of each source. Objects which had only a single association within 3 arcmin were further matched with a closer radius of 30 arcsec and if the radio association was still found within this radius the source was classified as NVSS single. For objects with multiple matches within 3 arcmin we extracted maps of $0.45 \mathrm{deg} \times 0.45 \mathrm{deg}$ in size from the NVSS Postage Stamp Service 1 and visually inspected whether associations found by us actually do or do not belong to the source. In order to do so we used the NRAO AIPS (Astronomical Image Processing System) package and NED (NASA/IPAC Extragalactic Database $\sqrt{2}$ which allowed us to distinguish incorrect matches. Additionally, when the radio morphology was extended beyond the matching radius of 3 arcmin, the radius was gradually increased by 1 arcmin as long as the associations for the whole structure were found. The flux associated with all the matches contributing to the given source was totalled up and assigned to each object in our sample.

Since SUMSS has a similar resolution to NVSS, searching for radio associations in this catalogue followed the same procedure as adopted for NVSS catalogue with radio maps extracted from the SUMSS Postage Stamp Download Data given in this survey provides fluxes at $843 \mathrm{MHz}$ which were re-calibrated to $1.4 \mathrm{GHz}$ using $\alpha_{\text {radio }}=0.8$ (with the convention $F_{v} \propto v^{-\alpha}$, e.g. Blumenthal \& Miley 1979, Kimball \& Ivezić 2008).

\footnotetext{
1 https://www.cv.nrao.edu/nvss/postage.shtml

2 https://ned.ipac.caltech.edu/

3 http://www.astrop.physics.usyd.edu.au/cgi-bin/postage.pl
} 


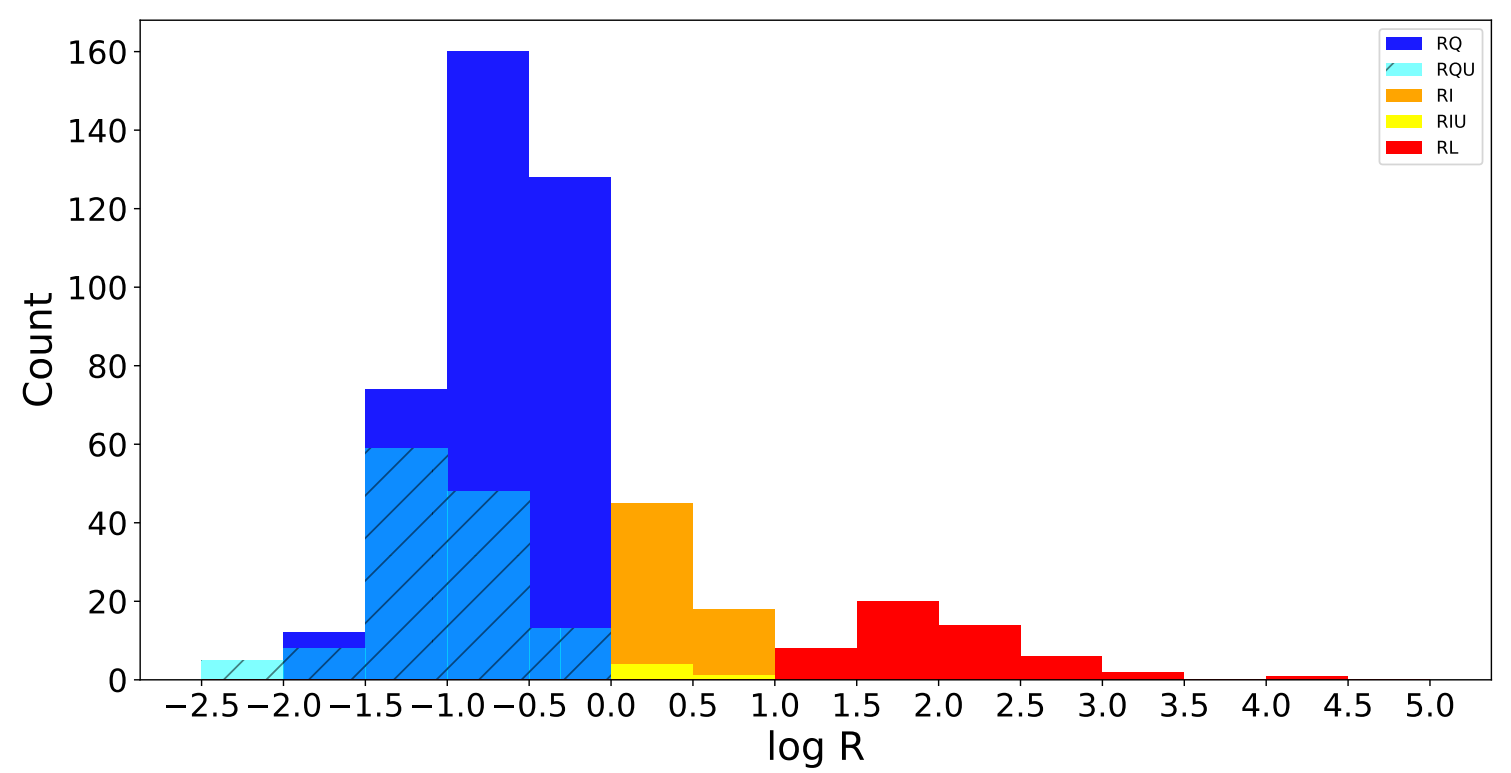

Figure 1. The distribution of the radio loudness, $R=F_{1.4} / F_{\nu_{\mathrm{W}} 3}$, for our sample. Besides showing objects with radio detections and categorized as: $R<1$ as radio-quiet (RQ); $1<R<10$ as radio-intermediate (RI); and $R>10$ as radio-loud (RL), we also present sources named as radio undetected (RQU and RIU).

\subsection{Mid-infrared data}

In order to obtain the infrared data for the sources in our sample we used the AllWISE Data Release (Cutri et al. 2013). The AllWISE release builds upon the work of the Wide-field Infrared Survey Explorer mission (WISE; Wright et al. 2010) which observed the sky at 3.4, 4.6, 12 and 22 $\mu \mathrm{m}$ ( $W 1, W 2, W 3$ and $W 4$ band, respectively) and combines it with the data from the WISE cryogenic and NEOWISE (Mainzer et al.2011) post-cryogenic survey phases giving the best view of the mid-infrared (MIR) sky available till date. For determining the counterparts for our sources we decided on 5 arcsec as the matching radius which arises from the fact that the angular resolution of WISE is $6.1,6.4,6.5$ and 12.0 arcsec in the four bands, respectively. Using a larger matching radius would result in duplicate WISE sources around our AGNs and higher rate of false positives in associations.

In order to accomplish our goal to use MIR data to determine radio loudness and bolometric luminosities regardless the optical type of AGNs we decided to use only data from the $W 3$ band. That is because at shorter wavelengths (those covered by $W 1$ and $W 2$ bands) the dusty, circumnuclear tori are optically thick and are radiating anisotropically (Hönig et al. 2011 Netzer 2015), while measurements in W4 band are affected by much larger errors than in $W 3$ band. The monochromatic flux, $F_{\nu_{\mathrm{W} 3}}$, is calculated using relation $\log F_{v_{\mathrm{W} 3}}=-\left(53.774+m_{\mathrm{W} 3}\right) / 2.5$, where $m_{\mathrm{W} 3}$ is the magnitude provided in the WISE catalogue.

\subsection{Constructing the RL and RQ samples}

The radio loudness parameter is defined by us as $R=$ $F_{1.4} / F_{\nu_{\mathrm{W} 3}}$, where $F_{1.4}$ and $F_{\nu_{\mathrm{W} 3}}$ are the monochromatic fluxes at $1.4 \mathrm{GHz}$ and $2.5 \times 10^{13} \mathrm{~Hz}$, respectively. This is re- lated to the definition given by Kellermann et al. (1989) as $R_{\mathrm{KL}}=F_{5} / F_{\gamma_{\mathrm{B}}}$, where $F_{5}$ and $F_{\gamma_{\mathrm{B}}}$ are the monochromatic fluxes at $5 \mathrm{GHz}$ and $6.8 \times 10^{14} \mathrm{~Hz}$, respectively. Both these quantities are linked by assuming radio spectral index $\alpha_{1.4-5}=0.8$ and $\alpha_{\nu_{\mathrm{B}}-\nu_{\mathrm{W} 3}}=1$ (Richards et al. 2006), and with them the relation between our radio loudness $R$ and the Kellerman parameter $R_{\mathrm{KL}}$ is found as $R \approx 0.1 \times R_{\mathrm{KL}}$.

Using the division corresponding to that based on the Kellermann et al. (1989) definition of radio loudness we define the following subsets in our sample: $R<1$ as RQ; $1<R<10$ as radio-intermediate (RI); and $R>10$ as RL. The distribution of the radio loudness of the sources in our sample is shown in Fig 1 Together with RQ, RI and RL subsamples we present $\mathrm{RQ}$ and $\mathrm{RI}$ sources for which the radio associations were not found. They are named as RQU and RIU objects (with the radio flux as $2.5 \mathrm{mJy}$ ), respectively.

We hereafter reject from our further analysis RI AGNs and those radio-undetected objects which have radio loudness upper limits $>1$. This assures of having two 'clean' subsamples, those with $R>10$ where radio emission is expected to be dominated by jets, and those with $R<1$, where the total radio emission flux is likely to be dominated by star-forming regions (e.g. Kimball et al. 2011), accretion disc winds (e.g. Zakamska et al. 2016), and/or accretion disc coronae (e.g. Raginski \& Laor 2016). The resulting source count is 51 RL AGNs and 509 RQ AGNs.

\section{$3 S W I F T / B A T$ AGNS IN $M_{\mathrm{BH}}-\lambda_{\mathrm{E}}$ PLANE}

\subsection{The $\mathrm{BH}$ masses and Eddington ratios}

Black hole masses for the objects in our sample were taken from Koss et al. (2017). For AGN type 1 they were obtained using broad emission lines $\mathrm{H} \beta$, for AGN type 2 - using stellar 


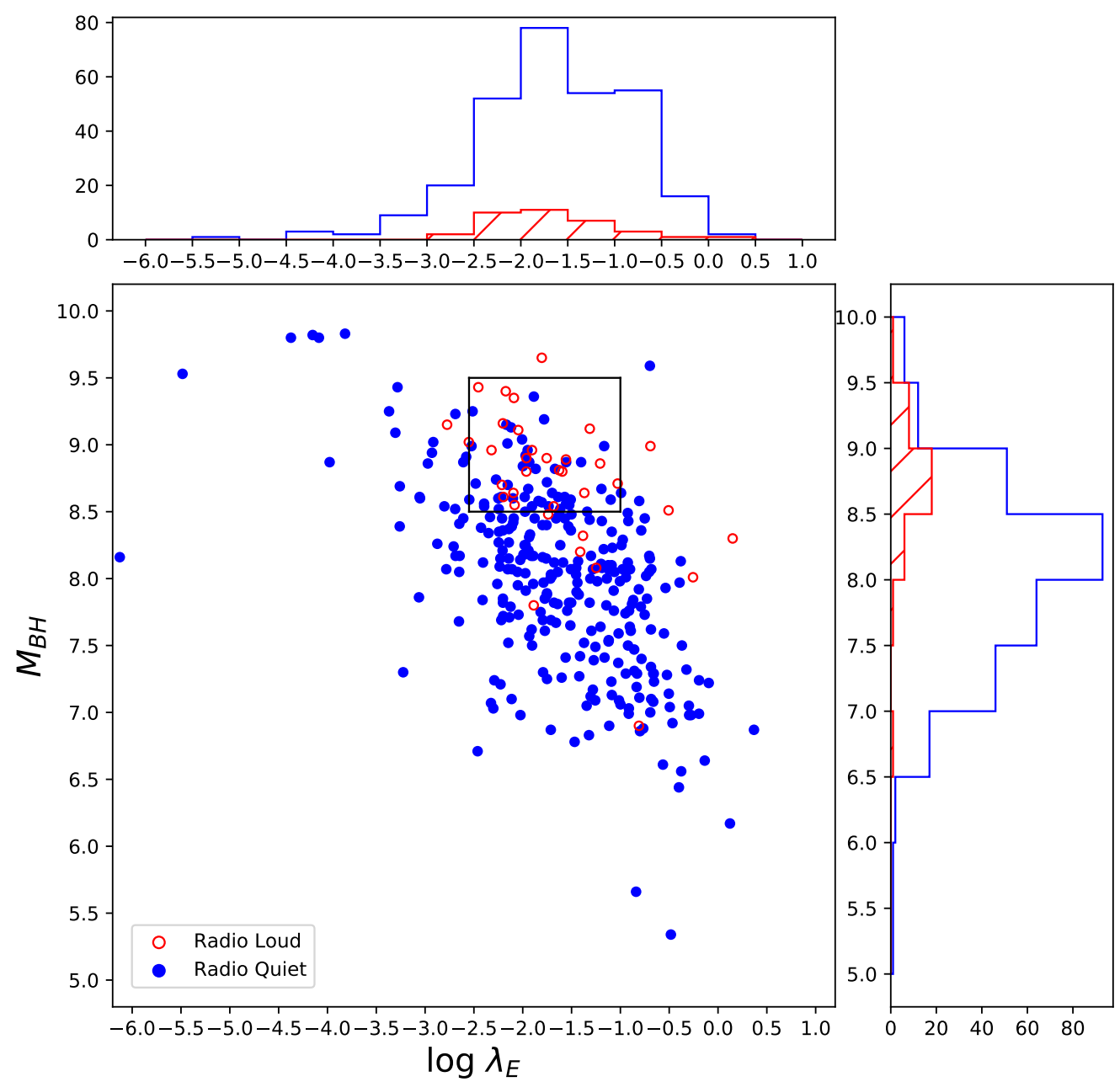

Figure 2. The distribution of the black hole masses, $M_{\mathrm{BH}}$, and Eddington ratios, $\lambda_{\mathrm{E}}$, for RL (empty red) and RQ (filled blue) objects in our sample. The inlaid box represents the ranges of $M_{\mathrm{BH}}$ and $\lambda_{\mathrm{E}}$ cut-offs we focus on in this paper.

Table 1. Steps involved in construction of our sample.

\begin{tabular}{cc}
\hline Number of objects & Method \\
\hline \hline 838 & Sample from Ricci et al. $\sqrt{2017})$ \\
776 & Cut-off of $\log N_{H}<24$ \\
664 & Removing blazars \\
630 & Valid w3 magnitude \\
560 & Restricting our study to RL and RQ sources \\
328 & Valid $M_{\mathrm{BH}}$ estimation \\
$70(24 \mathrm{RL}, 46 \mathrm{RQ})$ & Cut-off of $8.48 \leq \log M_{\mathrm{BH}} \leq 9.5$ and $-2.55 \leq \log \lambda_{\mathrm{E}} \leq-1$ \\
\hline
\end{tabular}

velocity dispersion. For some AGNs in our sample lacking information on BH masses in Koss et al. (2017) we took them from Panessa et al. (2015). Together we gathered BH mass data for 328 AGNs (293 for RQ and 35 for RL).

Koss et al. (2017) derived also bolometric luminosities. They assumed that bolometric luminosity is traced by the hard X-ray luminosities with respective bolometric correction. Noting however, that previous studies indicated that RL AGNs are X-ray-louder than RQ AGNs, we decided to use as a tracer of bolometric luminosity the MIR luminosi- ties (see e.g. Runnoe et al. 2012, Fernandes et al.|2015). This choice can be justified by noting: (i) that the MIR emission in AGNs is dominated by dust heated by central engine (e.g. Mateos et al. 2015 Ricci et al. 2017, Hönig \& Kishimoto 2017; Zhuang et al. 2018); (ii) that the 'effective' covering factors of dusty "obscurers" (circumnuclear tori/winds), defined to be the fraction of the optical/UV radiation converted by dust to the IR radiation, are very similar in RL and RQ AGNs (Ma \& Wang 2013, Gupta et al. 2016, Hickox et al. 2017) and (iii) that at $\lambda>10 \mu \mathrm{m}$ the AGN dusty obscur- 
ers are optically thin and radiate isotropically (e.g. Mateos et al. 2015).

The bolometric luminosity calculated by us is $L_{\mathrm{bol}}=$ $K_{\mathrm{W} 3} \nu_{\mathrm{W} 3} L_{\mathcal{V}_{\mathrm{W} 3}}$, where $L_{\mathcal{V}_{\mathrm{W} 3}}=4 \pi d_{I}^{2} F_{\nu_{\mathrm{W} 3}} /(1+z)^{1-\alpha_{\mathrm{IR}}}$ and $K_{\mathrm{W} 3}=$ 8.4, where $\lambda_{W 3}=12 \mu \mathrm{m}$ (Richards et al. 2006). Since dependence of this formula on $\alpha_{\mathrm{IR}}$ for small redshifts is very weak, we adopted $\alpha_{\mathrm{IR}}=1$ (Mateos et al. 2015). And combining this with $\mathrm{BH}$ masses and definition of Eddington luminosity we obtain Eddington ratios, $\lambda_{E}=L_{\mathrm{bol}} / L_{\mathrm{Edd}}$.

Distribution of AGNs in the plane ' $M_{\mathrm{BH}}-\lambda_{E}$ ' is shown in Fig. 2 As we can see, there is a deficiency of AGNs in the upper-right corner of the figure and in the lower-left corner of the figure. The low representation of AGNs at large Eddington ratios and large $\mathrm{BH}$ masses can be explained as resulting from the steep mass function of AGN black holes (see review by Kelly \& Merloni 2012) and downsizing effect (Fanidakis et al. 2012), while poor representation of AGNs at low Eddington ratios and small BH masses is associated with the flux limit of Swift/BAT survey. Furthermore, we can see very poor representation of RL AGNs with BH masses smaller than $10^{8} M_{\odot}$ at any Eddington ratio. Such a dependence of radio loudness on BH mass, albeit well documented ( McLure \& Jarvis 2004, Koziel-Wierzbowska et al. 2017 and refs. therein), is still lacking consensus regarding its explanation (see e.g. Chiaberge \& Marconi 2011, Sikora \& Begelman 2013 Cao 2016). The objects at $\lambda_{\mathrm{E}}<10^{-5}$ should not be considered here, since the method applied by us for deriving bolometric luminosity is expected to work only in the regime of radiatively efficient accretion flows.

\subsection{Luminous RGs and their 'close' RQ counterparts - the GSRM sample}

Hereafter we focus on the RL and RQ AGNs covering similar ranges of $\mathrm{BH}$ masses and Eddington ratios and restrict our studies to AGNs with $8.5 \leq \log M_{\mathrm{BH}} \leq 9.5$ and $-2.55 \leq$ $\log \lambda_{\mathrm{E}} \leq-1$. This subset of AGNs is marked in Fig. 2 by an inlaid box and is named by us the GSRM sample. It includes 70 objects consisting of $24 \mathrm{RL}$ and $46 \mathrm{RQ}$ AGNs, and detailed data on those objects are provided in Table 6 Their redshift distributions are shown in Fig. 3 We see a significant overlap between them, and their median values are 0.084 for RL AGNs and 0.070 for RQ AGNs.

Distributions of radio loudness, $R$, of the GSRM RQ and RL subsamples are presented in Fig. 4. The median value of the radio loudness of $R L$ bjects is 122 and of $R Q$ objects is by a factor $\sim 500$ smaller.

For each of the sources in our sample, we extracted radio maps from NVSS and SUMSS surveys (as described in section 2.2. We found that most of RL AGNs in the GSRM sample have FR II radio morphology. 20 of radio galaxies in the GSRM sample were also identified with Swift/BAT AGNs by Bassani et al. (2016). Among them we can distinguish three FR II objects with peculiar radio structure: SWIFT J2359.3-6058 being a giant radio galaxy (GRG, Malarecki et al. 2015); SWIFT J2223.9-0207 which is a wellaligned double-double giant radio galaxy (DDRG, Saikia et al. 2006); and one X-shaped source, SWIFT J1952.4+0237 (Capetti et al. 2002). Four GSRM RL AGNs are not identified by Bassani et al. They are: SWIFT J0021.2-1909, which is a triple in NVSS; SWIFT J0235.3-2934 with two radio matches in NVSS and extended (but not FR II) structure;

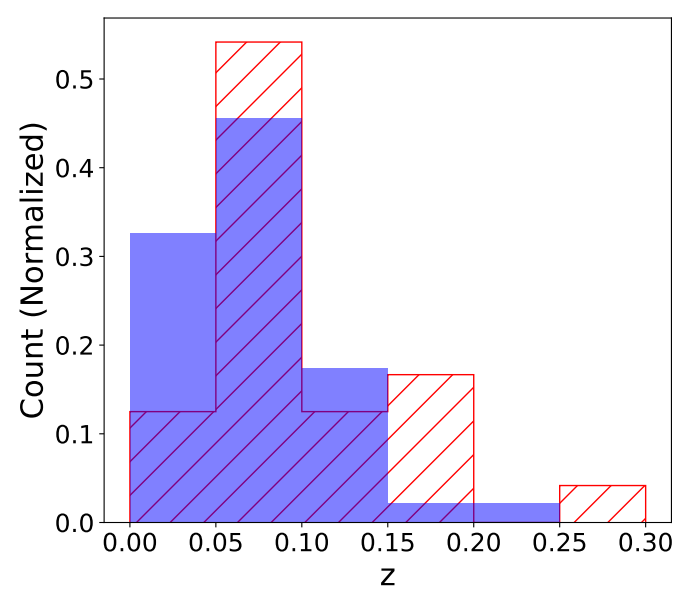

Figure 3. The normalized distribution of the redshift of RL (hatched red) and RQ (filled blue) samples of GSRM AGNs.

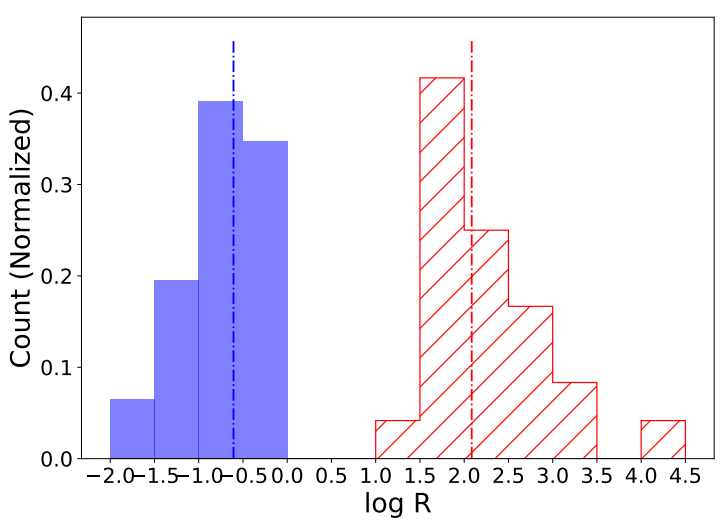

Figure 4. The normalized distribution of RL (hatched red) and RQ (filled blue) subsamples of GSRM AGNs in radio loudness. The median values are presented as dashed lines for both samples.

and SWIFT J1207.5+3355 and SWIFT J2351.9-0109 - both found as singles on NVSS map and probably FR II on FIRST (Faint Images of the Radio Sky at Twenty-centimeters; Becker et al. 1995 maps. Detailed studies of radio sources associated with GSRM AGNs will be investigated in our future paper, where the issue of radio bimodality will be addressed.

It is worth mentioning here that the number ratio of $\mathrm{RL}$ to RQ objects in the GSRM sample is $\sim 1: 2$. This ratio is much larger than the ratio $\sim 1: 10$ of $\mathrm{RL}$ quasars to RQ quasars (de Vries et al. 2006). This difference can be explained by noting that in our sample all AGNs host very massive $\mathrm{BHs}$ and that the fraction of RL AGNs among AGNs with $M_{\mathrm{BH}}>10^{8} M_{\odot}$ is larger than among AGNs with smaller BH masses (see e.g. McLure \& Jarvis 2004). However, one cannot exclude a possibility that the fraction of the RL AGNs depends also on the Eddington ratio being larger at lower accretion rates (see Sikora et al. 2007, Rusinek et al. 2017 and references therein).

Fig. 5 shows the distribution of column density, $N_{H}$, for 


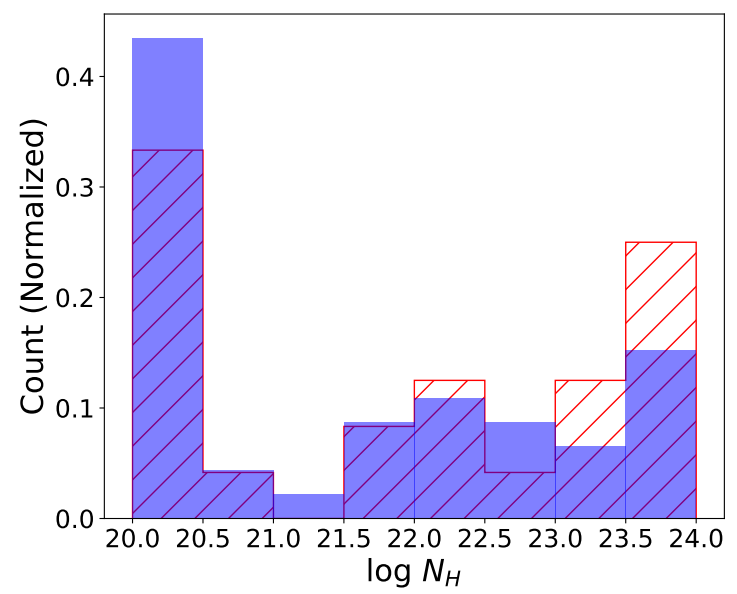

Figure 5. The normalized distribution of the column density, $N_{\boldsymbol{H}}$, of RL (hatched red) and RQ (filled blue) samples of GSRM AGNs.

the RL and RQ samples. We performed the KolmogorovSmirnov test to the distributions of column density for the RL and RQ samples and observe that they are very similar (see Table 2). This indicates similar covering factors of circumnuclear dusty obscurers and validates our method of using MIR to determine the bolometric luminosity with the same bolometric correction for RL and RQ AGNs (see also previous subsection). However it should be noted that at $\log N_{H}<20.5$ the column densities are dominated by the Milky Way and host galaxies rather than by the AGN circumnuclear obscurers.

\section{COMPARISON OF X-RAY PROPERTIES}

\subsection{The X-ray loudness}

The definition of X-ray loudness for our sample is adopted as the ratio of hard X-ray luminosity to bolometric luminosity, $L_{14-195} / L_{\text {bol }}$. Distributions of X-ray loudness for RL and RQ AGNs are presented in Fig. 6. As we can see the X-ray loudness distribution of RQ AGNs is broader and extends to much lower values than the distribution of RL AGNs. The median value of X-ray loudness is $\sim 0.4$ and $\sim 0.2$. for RL and RQ sources, respectively. Therefore we observe that the RL objects are on average about two times X-ray-louder than their RQ counterparts in the $14-195 \mathrm{keV}$ band. The low p-value of 0.0007 of the K-S test indicates that the Xray loudness distribution for the RL and RQ samples are quite different (see Table 2). This difference could be larger by considering the possibility of greater incompleteness of the X-ray-detected RQ AGNs than of RL AGNs. However location of members of the GSRM sample in the $L_{14-195^{-}}$ redshift plane relative the flux-limited luminosity curve (see Fig. 7) indicates that within the ranges of $\mathrm{BH}$ masses and Eddington ratios covered by this sample this incompleteness is rather negligible.

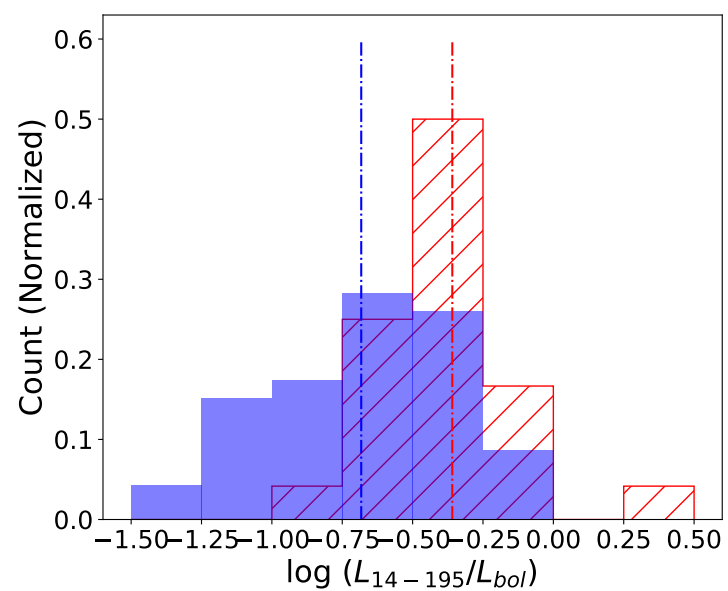

Figure 6. The normalized distribution of RL (hatched red) and RQ (filled blue) subsamples of GSRM AGNs in X-ray loudness which is defined as the ratio of hard X-ray luminosity to bolometric luminosity. The median values are presented as dashed lines for both samples.

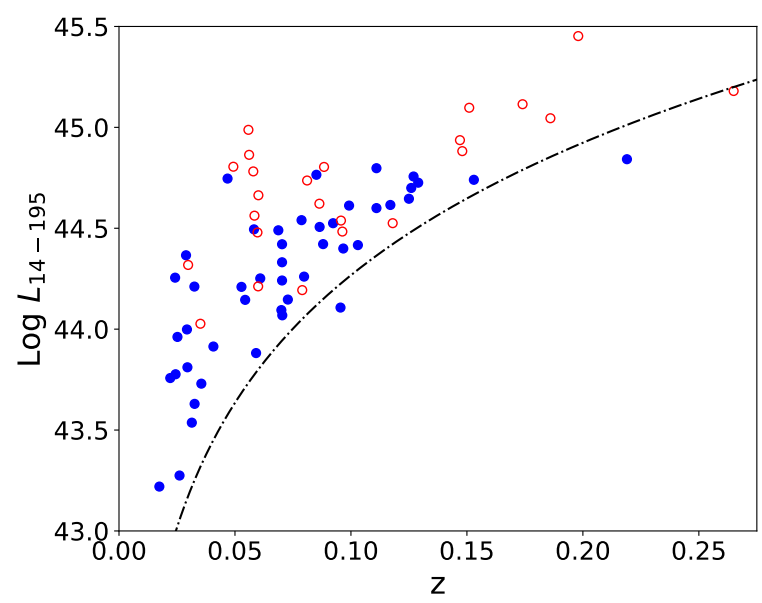

Figure 7. The distribution of hard X-ray luminosity versus redshift of RL (empty red) and RQ (filled blue) subsamples of GSRM AGNs.

\subsection{The spectral slopes}

In our study we decided on using the photon index $\Gamma_{14-195}$ values provided by Ricci et al. (2017) instead of those from BAT sample (Baumgartner et al. 2013) where the obscuration by dusty torus was not taken into account. The distribution of $\Gamma_{14-195}$ for the RL and RQ sources is shown in Fig. 8 We clearly see a significant overlap between both samples with very similar median values being 1.740 and 1.735 for $\mathrm{RL}$ and RQ sources respectively and the high p-value of 0.969 of the K-S test indicates that the spectral index distribution for the RL and RQ samples is very similar (see Table 2).

Even though in many previous studies the emission of hard X-rays was found to be higher for RL than RQ AGNs (e.g. Zamorani et al. 1981, Kataoka et al. 2011; Worrall et al. 1987. Woźniak et al. 1998, Miller et al. 2011, and references 


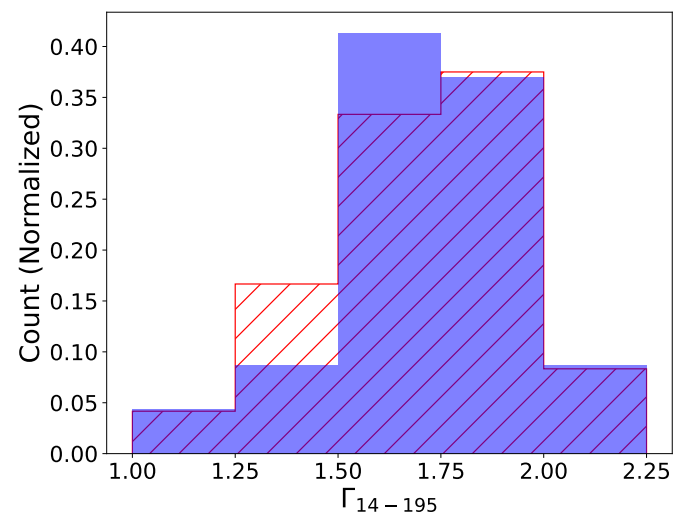

Figure 8. The normalized distribution of the photon index, $\Gamma_{14-195}$, of RL (hatched red) and RQ (filled blue) subsamples of GSRM AGNs.

Table 2. Results of the Kolmogorov-Smirnov test performed on the distributions of column density, X-ray loudness, and spectral slope for the RL and RQ samples

\begin{tabular}{ccc}
\hline Distribution & $D$ & $p$-value \\
\hline \hline $\log N_{H}$ & 0.190 & 0.571 \\
$\log L_{14-195} / L_{\text {bol }}$ & 0.486 & 0.0007 \\
$\Gamma_{14-195}$ & 0.119 & 0.969 \\
\hline
\end{tabular}

therein) until now none of them has focused on similar black hole mass and Eddington ratio ranges. While considering this aspect one can notice no difference in the photon index distribution between RL and RQ samples which suggests a similar underlying mechanism in the production of X-rays being independent on the radio loudness value.

\subsection{High-energy cut-offs and reflection features}

The high-energy cut-off values were, in similarity to the photon gamma index, taken from Ricci et al. (2017). Some of the sources have given only the lower limits while for three RL and three RQ sources there are no data resulting in excluding them in this analysis. The distributions of high-energy cut-off for RL and RQ subsamples are presented in Fig. 9a and $9 \mathrm{~b}$, respectively, and they are comparable. Having the high-energy break for RL and RQ AGNs around the same place implies that the mechanism that produces the $\mathrm{X}$-rays is similar.

Regarding the reflection features, we also use results obtained by Ricci et al. (2017). Those authors provide constraints on the reflection parameter $R_{\text {refl }}=\Omega / 2 \pi$, where $\Omega$ is the solid angle subtended by the reflecting material, calculated using data from Swift/XRT and other X-ray telescopes covering the X-ray bands below $10 \mathrm{keV}$. Values of $R_{\text {refl }}$ are found for two RL AGNs and nine RQ AGNs, for others only upper limits are available (see Fig. 10a and Fig. 10b). The larger fraction of RQ AGNs with determined values of $R_{\text {refl }}$ than of RL AGNs (9/46 in RQ AGNs versus 2/24 in RL AGNs) and larger on average upper limits of $R_{\text {refl }}$ in RQ AGNs indicate smaller on average covering factor of reflect- ing material in RL AGNs. This is in agreement with results obtained by other studies of reflection features disregarding different selection methods of RL and RQ AGNs (e.g. Woźniak et al. 1998, Miller et al. 2011, and references therein).

\section{DISCUSSION}

Perhaps the largest puzzle in our understanding of active galaxies still remains the very large diversity of their jet activity. This is particularly most apparent in AGNs with higher accretion rates, where power of a jet traced by the radio luminosity can be compared with accretion power traced by optical luminosity of accretion discs. At each Eddington ratio the radio loudness spans at least by three to four orders of magnitude (e.g. Sikora et al. 2007). Jets are observed also in some RI and RQ AGNs (see e.g. Smith et al. 2016 and references therein), but in many (probably most) of them the radio emission is likely to be dominated by star formation regions (SFR e.g. Kimball et al. 2011), the shocked accretion disc winds (e.g. Zakamska et al. 2016), and/or accretion disc coronae (e.g. Raginski \& Laor 2016). Hence the range of radio luminosities associated with the jet activity is larger than the one determined assuming that even in RQ AGNs radio emission is dominated by jets. And regardless whether the jets are present and how powerful they are, quasar SEDs covering IR, optical and UV bands are very similar, and statistically significant differences appear only in X-ray bands (Elvis et al. 1994, Richards et al. 2006, Shang et al. 2011). In particular the RL quasars are found to have on average: (1) larger X-ray-to-optical luminosity ratios; (2) harder X-ray spectra; (3) weaker reflection features. Similar differences have been claimed for lower redshift type 1 AGNs, those represented by broad-line radio galaxies and RQ type 1 Seyferts and having on average lower Eddington ratios than quasars (Miller et al. 2011, Kataoka et al. 2011). In most of these studies the compared RL and RQ samples were selected independently, without considering the differences of their average black hole masses and Eddington ratios. Since $\mathrm{X}$-ray properties may depend on these parameters, we decided to compare X-ray properties of RLAGNs with their RQ counterparts selecting them to cover a similar range of $\mathrm{BH}$ masses and accretion rates, namely $8.5 \leq \log M_{\mathrm{BH}} \leq 9.5$ and $-2.55 \leq \log \lambda_{\mathrm{E}} \leq-1$.

We found that indeed RQ counterparts of luminous radio galaxies have on average lower X-ray luminosities, but only by a factor $\sim 2$. On the other hand, similar distributions of hard X-ray spectral slopes and of high-energy spectral cuto-ffs of RL and RQ GSRM subsamples which in radio loudness differ by a factor $\sim 500$ implies that production of even very powerful jets does not affect significantly the properties of the primary X-ray sources. This strongly suggests similar mechanism and location of hard X-ray production in RQ and RL AGNs and therefore appears to exclude any significant contribution of jets to the X-ray luminosities even in RL AGNs. A scenario favoured by detailed observations and studies of individual BLRGs (e.g. Eracleous et al. 2000, Grandi \& Palumbo 2007, Sambruna et al. 2009, Tazaki et al. 2010 Lohfink et al. 2013) seems to have hard X-ray emission result from Comptonization of optical/UV radiation produced by cold, truncated accretion disc by high-energy electrons in hot, geometrically thick ADAFs between the 


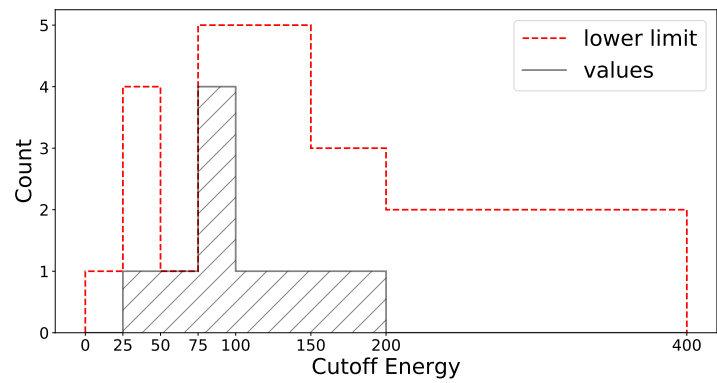

(a)

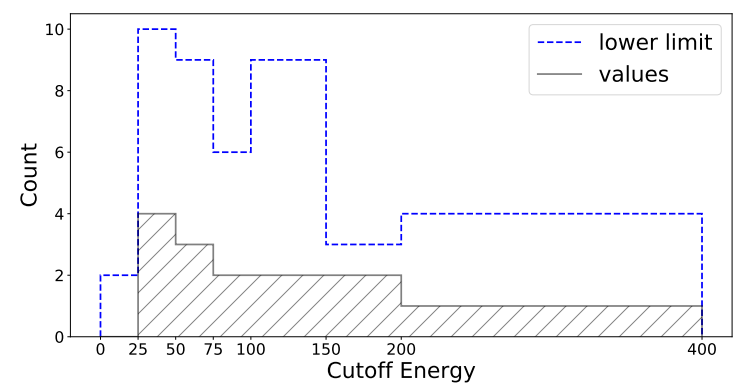

(b)

Figure 9. Cut-off energy distribution. The plots show the values (black solid histogram) and the lower limits (dashed histogram). (a) Distribution of the cut-off energy for the RL AGNs of GSRM sample. (b) Distribution of the cut-off energy for the RQ AGNs of GSRM sample.

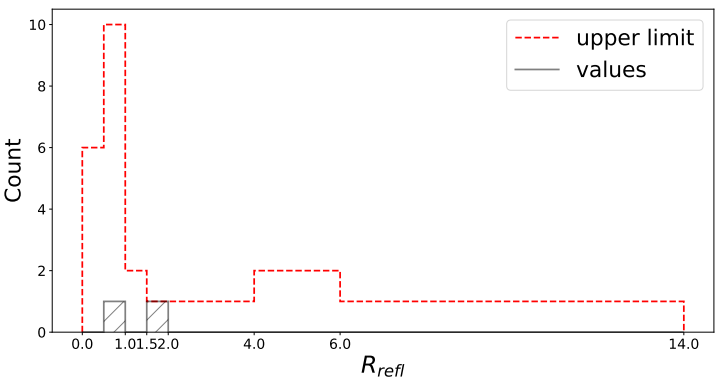

(a)

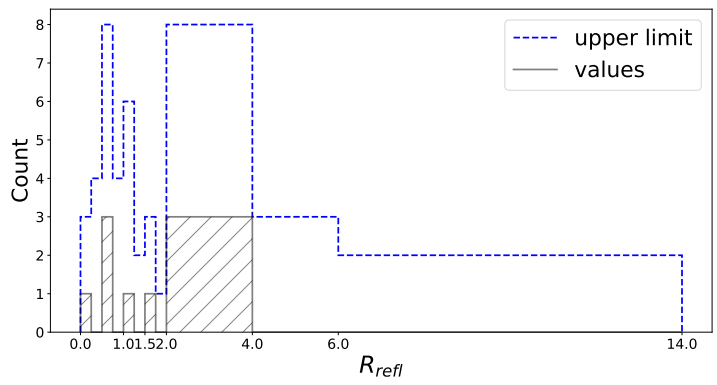

(b)

Figure 10. Reflection coefficient distribution. The plots show the values (black solid histogram) and the upper limits (dashed histogram). (a) Distribution of the reflection parameters for the RL AGNs of GSRM sample. (b) Distribution of the reflection parameters for the RQ AGNs of GSRM sample.

cold disc and the black hole (for more complex structures of thermal Compton models of X-ray production in AGNs see e.g. Kubota \& Done 2018 and references therein). In such models the high-energy cut-offs of the X-ray spectra are determined by temperature of electrons heated in ADAFs by hotter ions.

The GSRM sample selected by us is planned to be used for more detailed comparisons of RL and RQ AGNs, including detailed radio data, narrow emission lines, optical properties of their host galaxies, environments, and in case of AGN type 1, their SEDs covering NIR, optical, UV and soft-X-ray bands.

\section{SUMMARY}

By matching the Swift/BAT catalogue with the NVSS and SUMSS catalogues we selected RL and RQ samples of AGNs having detection in the $14-195 \mathrm{keV}$ band. Then, estimating their bolometric luminosities using mid-IR data from WISE and taking BH masses from Koss et al. (2017) and for some RL AGNs not included in the paper by Koss et al. from Panessa et al. (2015), we selected two respective subsamples covering similar ranges of $\mathrm{BH}$ masses and Eddington ratios, $8.5 \leq \log M_{\mathrm{BH}} \leq 9.5$ and $-2.55 \leq \log \lambda_{\mathrm{E}} \leq-1$. They are represented by 24 luminous radio galaxies and by 46 RQ AGNs. Both subsamples have similar X-ray spectral slopes. They have also similar maximal values of X-ray loudness, however with RQ AGNs having lower average X-ray loudness than radio galaxies. Above similarities suggest that the location and mechanism of hard X-ray production are the same in radio galaxies and in their RQ counterparts. The mechanism most likely involves Comptonization of optical/UV radiation of truncated cold accretion disc by hot electrons in centrally developed ADAFs. In this scenario the larger on average X-ray loudness of RL AGNs than of RQ ones can be explained assuming that BHs in RL AGNs rotate faster. This is because in such a case efficiency of conversion of accretion energy to radiation in hot central portions of accretion flows is expected to be larger. Furthermore, in a case of larger BH spins in radio louder AGNs one may expect to have in such objects larger central concentration of the hard X-ray emission, which in turn may explain lower reflection features in luminous radio galaxies than in their $\mathrm{RQ}$ counterparts.

\section{ACKNOWLEDGEMENTS}

We acknowledge financial support by the Polish National Science Centre grants 2016/21/B/ST9/01620 and 2017/25/N/ST9/01953, GM acknowledges the support of the United States Department of Energy Office of Science contract to SLAC no. DE-AE3-76SF00515. 


\section{REFERENCES}

Ballantyne, D. R., Ross, R. R., \& Fabian, A. C. 2002, MNRAS, $332, \mathrm{~L} 45$

Ballantyne, D. R., Bollenbacher, J. M., Brenneman, L. W., et al. 2014, ApJ, 794, 62

Baloković, M., Smolčić, V., Ivezić, Ž., et al. 2012, ApJ, 759, 30

Bassani, L., Venturi, T., Molina, M., et al. 2016, MNRAS, 461, 3165

Baumgartner, W. H., Tueller, J., Markwardt, C. B., et al. 2013, ApJS, 207, 19

Becker, R. H., White, R. L., \& Helfand, D. J. 1995, ApJ, 450, 559

Blandford, R. D., \& Znajek, R. L. 1977, MNRAS, 179, 433

Blumenthal, G., \& Miley, G. 1979, A\&A, 80, 13

Bock, D. C.-J., Large, M. I., \& Sadler, E. M. 1999, AJ, 117, 1578

Cao, X. 2016, ApJ, 833, 30

Capetti, A., Zamfir, S., Rossi, P., et al. 2002, A\&A, 394, 39

Chiaberge, M., \& Marconi, A. 2011, MNRAS, 416, 917

Condon, J. J., Cotton, W. D., Greisen, E. W., et al. 1998, AJ, 115, 1693

Cowperthwaite, P. S., \& Reynolds, C. S. 2012, ApJ, 752, L21

Cutri, R. M., et al. 2013, VizieR Online Data Catalog, 2328,

de Vries, W. H., Becker, R. H., \& White, R. L. 2006, AJ, 131, 666

Elvis, M., Wilkes, B. J., McDowell, J. C., et al. 1994, ApJS, 95, 1

Eracleous, M., Sambruna, R., \& Mushotzky, R. F. 2000, ApJ, 537, 654

Evans, D. A., Reeves, J. N., Hardcastle, M. J., et al. 2010, ApJ, 710,859

Fanaroff, B. L., \& Riley, J. M. 1974, MNRAS, 167, 31P

Fanidakis, N., Baugh, C. M., Benson, A. J., et al. 2012, MNRAS, 419, 2797

Fernandes, C. A. C., Jarvis, M. J., Rawlings, S., et al. 2011, MNRAS, 411, 1909

Fernandes, C. A. C., Jarvis, M. J., Martínez-Sansigre, A., et al. 2015, MNRAS, 447, 1184

Grandi, P., Malaguti, G., \& Fiocchi, M. 2006, ApJ, 642, 113

Grandi, P., \& Palumbo, G. G. C. 2007, ApJ, 659, 235

Gupta, M., Sikora, M., \& Nalewajko, K. 2016, MNRAS, 461, 2346

Haardt, F., \& Maraschi, L. 1991, ApJ, 380, L51

Hickox, R. C., Myers, A. D., Greene, J. E., et al. 2017, ApJ, 849, 53

Hönig, S. F., Leipski, C., Antonucci, R., \& Haas, M. 2011, ApJ, 736,26

Hönig, S. F., \& Kishimoto, M. 2017, ApJ, 838, L20

Igumenshchev, I. V. 2008, ApJ, 677, 317

Ivezić, Ž., Menou, K., Knapp, G. R., et al. 2002, AJ, 124, 2364

Kataoka, J., Stawarz, Ł., Takahashi, Y., et al. 2011, ApJ, 740, 29

Kellermann, K. I., Sramek, R., Schmidt, M., Shaffer, D. B., \& Green, R. 1989, AJ, 98, 1195

Kelly, B. C., \& Merloni, A. 2012, Advances in Astronomy, 2012, 970858

Kimball, A. E., \& Ivezić, Ž. 2008, AJ, 136, 684

Kimball, A. E., Kellermann, K. I., Condon, J. J., Ivezić, Ž., \& Perley, R. A. 2011, ApJ, 739, L29

Koss, M., Trakhtenbrot, B., Ricci, C., et al. 2017, ApJ, 850, 74

Kozieł-Wierzbowska, D., Vale Asari, N., Stasińska, G., et al. 2017, ApJ, 846, 42

Kratzer, R. M., \& Richards, G. T. 2015, AJ, 149, 61

Kubota, A., \& Done, C. 2018, MNRAS, 480, 1247

Liang, E. P. T., \& Thompson, K. A. 1979, MNRAS, 189, 421

Lohfink, A. M., Reynolds, C. S., Jorstad, S. G., et al. 2013, ApJ, 772,83

Ma, X.-C., \& Wang, T.-G. 2013, MNRAS, 430, 3445

Mainzer, A., Bauer, J., Grav, T., et al. 2011, ApJ, 731, 53

Malarecki, J. M., Jones, D. H., Saripalli, L., Staveley-Smith, L., \& Subrahmanyan, R. 2015, MNRAS, 449, 955

Massaro, E., Maselli, A., Leto, C., et al. 2015, Ap\&SS, 357, 75

Mateos, S., Carrera, F. J., Alonso-Herrero, A., et al. 2015, MN-
RAS, 449, 1422

Mauch, T., Murphy, T., Buttery, H. J., et al. 2003, MNRAS, 342, 1117

McKinney, J. C., Tchekhovskoy, A., \& Blandford, R. D. 2012, MNRAS, 423, 3083

McLure, R. J., \& Jarvis, M. J. 2004, MNRAS, 353, L45

Miller, L., Peacock, J. A., \& Mead, A. R. G. 1990, MNRAS, 244, 207

Miller, B. P., Brandt, W. N., Schneider, D. P., et al. 2011, ApJ, 726, 20

Narayan, R., \& Yi, I. 1994, ApJ, 428, L13

Narayan, R., Igumenshchev, I. V., \& Abramowicz, M. A. 2003, PASJ, 55, L69

Netzer, H. 2015, ARA\&A, 53, 365

Oh, K., Koss, M., Markwardt, C. B., et al. 2018, ApJS, 235, 4

Panessa, F., Tarchi, A., Castangia, P., et al. 2015, MNRAS, 447, 1289

Peacock, J. A., Miller, L., \& Longair, M. S. 1986, MNRAS, 218, 265

Punsly, B. 2007, MNRAS, 374, L10

Punsly, B., Igumenshchev, I. V., \& Hirose, S. 2009, ApJ, 704, 1065

Raginski, I., \& Laor, A. 2016, MNRAS, 459, 2082

Rawlings, S., \& Saunders, R. 1991, Nature, 349, 138

Ricci, C., Trakhtenbrot, B., Koss, M. J., et al. 2017, ApJS, 233, 17

Ricci, C., Trakhtenbrot, B., Koss, M. J., et al. 2017, Nature, 549, 488

Richards, G. T., Lacy, M., Storrie-Lombardi, L. J., et al. 2006, ApJS, 166, 470

Runnoe, J. C., Brotherton, M. S., \& Shang, Z. 2012, MNRAS, 426,2677

Rusinek, K., Sikora, M., Kozieł-Wierzbowska, D., \& Godfrey, L. 2017, MNRAS, 466, 2294

Saikia, D. J., Konar, C., \& Kulkarni, V. K. 2006, MNRAS, 366, 1391

Sambruna, R. M., Eracleous, M., \& Mushotzky, R. F. 1999, ApJ, 526,60

Sambruna, R. M., Reeves, J. N., Braito, V., et al. 2009, ApJ, 700, 1473

Shang, Z., Brotherton, M. S., Wills, B. J., et al. 2011, ApJS, 196, 2

Sikora, M., Stawarz, Ł., \& Lasota, J.-P. 2007, ApJ, 658, 815

Sikora, M., \& Begelman, M. C. 2013, ApJ, 764, L24

Smith, K. L., Mushotzky, R. F., Vogel, S., Shimizu, T. T., \& Miller, N. 2016, ApJ, 832, 163

Strittmatter, P. A., Hill, P., Pauliny-Toth, I. I. K., Steppe, H., \& Witzel, A. 1980, A\&A, 88, L12

Tazaki, F., Ueda, Y., Ishino, Y., et al. 2010, ApJ, 721, 1340

Tchekhovskoy, A., Narayan, R., \& McKinney, J. C. 2011, MNRAS, 418, L79

White, R. L., Helfand, D. J., Becker, R. H., Glikman, E., \& de Vries, W. 2007, ApJ, 654, 99

Wilkes, B. J., \& Elvis, M. 1987, ApJ, 323, 243

Worrall, D. M., Giommi, P., Tananbaum, H., \& Zamorani, G. 1987, ApJ, 313, 596

Wozniak, P. R., Zdziarski, A. A., Smith, D., Madejski, G. M., \& Johnson, W. N. 1998, MNRAS, 299, 449

Wright, E. L., Eisenhardt, P. R. M., Mainzer, A. K., et al. 2010, AJ, 140, 1868-1881

Yuan, F., \& Narayan, R. 2014, ARA\&A, 52, 529

Zakamska, N. L., Hamann, F., Pâris, I., et al. 2016, MNRAS, 459, 3144

Zamorani, G., Henry, J. P., Maccacaro, T., et al. 1981, ApJ, 245, 357

Zhuang, M.-Y., Ho, L. C., \& Shangguan, J. 2018, ApJ, 862, 118 


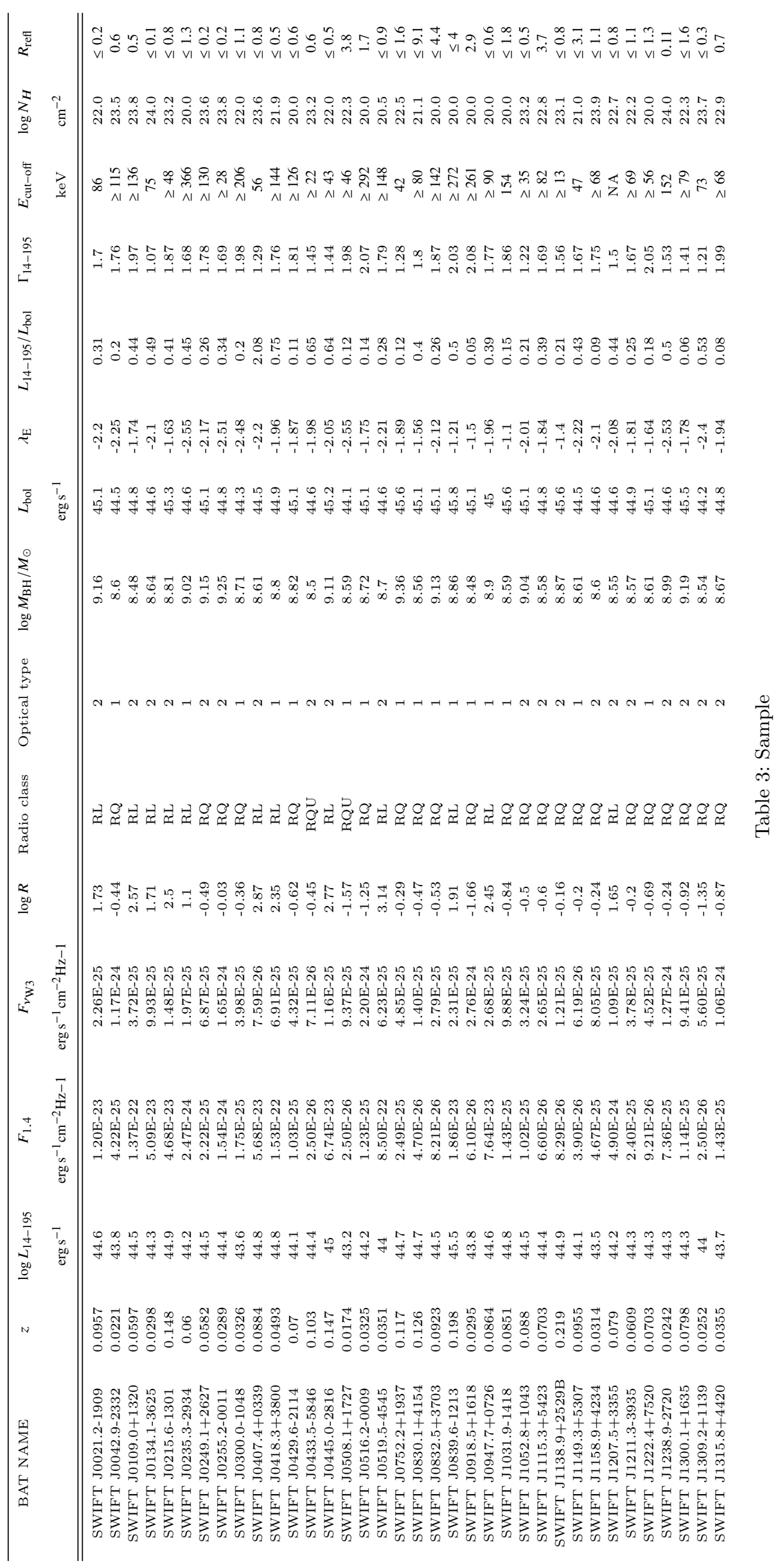

MNRAS 000,1 12(2015) 


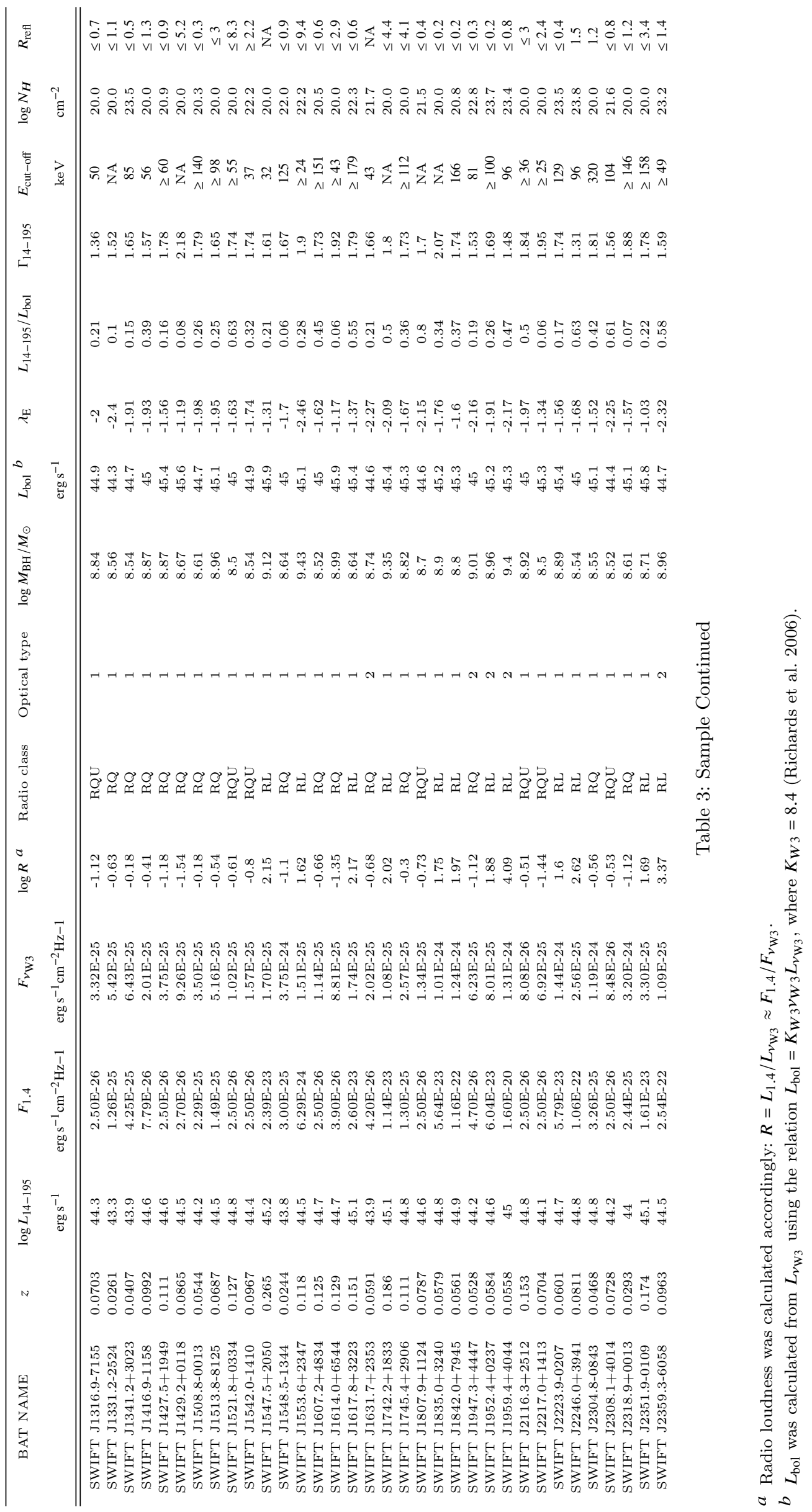




\section{Gupta et al.}

This paper has been typeset from a $\mathrm{T}_{\mathrm{E}} \mathrm{X} / \mathrm{LATEX}$ file prepared by the author. 